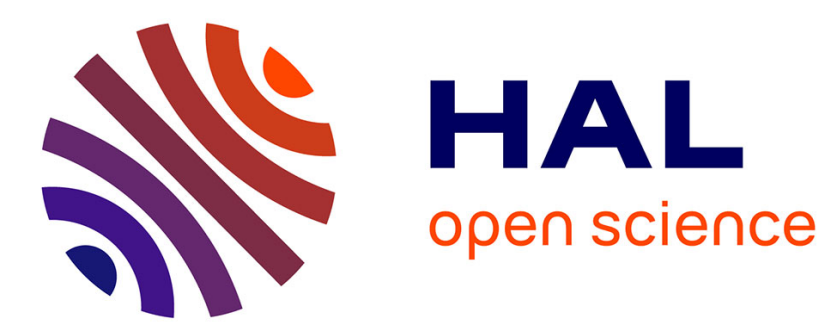

\title{
Structure of Salt-free Linear Polyelectrolytes in the Debye-Hückel Approximation
}

\author{
Mark Stevens, Kurt Kremer
}

\section{To cite this version:}

Mark Stevens, Kurt Kremer. Structure of Salt-free Linear Polyelectrolytes in the Debye-Hückel Approximation. Journal de Physique II, 1996, 6 (11), pp.1607-1613. 10.1051/jp2:1996151 . jpa00248396

\section{HAL Id: jpa-00248396 https://hal.science/jpa-00248396}

Submitted on 1 Jan 1996

HAL is a multi-disciplinary open access archive for the deposit and dissemination of scientific research documents, whether they are published or not. The documents may come from teaching and research institutions in France or abroad, or from public or private research centers.
L'archive ouverte pluridisciplinaire HAL, est destinée au dépôt et à la diffusion de documents scientifiques de niveau recherche, publiés ou non, émanant des établissements d'enseignement et de recherche français ou étrangers, des laboratoires publics ou privés. 


\title{
Structure of Salt-Free Linear Polyelectrolytes in the Debye-Hückel Approximation
}

\author{
Mark J. Stevens $\left({ }^{1}\right)$ and Kurt Kremer $\left({ }^{2, *}\right)$ \\ (') MS 1111, Sandia National Laboratory, Albuquerque, NM 87185, USA \\ $\left({ }^{2}\right)$ Max Planck Institut für Polymerforschung, Postfach 3148, 55021 Mainz, Germany
}

(Received 6 May 1996, revised 21 June 1996, accepted 31 July 1996)

\begin{abstract}
PACS.61.25.Hq - Macromolecular and polymer solutions; polymer melts; swelling
PACS.36.20.-r - Macromolecules and polymer molecules

PACS.87.15.-v - Molecular biophysics
\end{abstract}

\begin{abstract}
We examine the effects of the common Debye-Hückel approximation used in theories of polyelectrolytes. Molecular dynamics simulations using the Debye-Hückel pair potential of salt-free polyelectrolytes have been performed. The results of these simulations are compared to earlier "Coulomb" simulations which explicitly treated the counterions. We report here the comparisons of the osmotic pressure, the end-to-end distance and the single chain structure factor. In the dilute regime the Debye-Hückel chains are more elongated than the Coulomb chains implying that the counterion screening is stronger than the Debye-Hückel prediction. Like the Coulomb chains the Debye-Hückel chains contract significantly below the overlap density in contradiction to all theories. Entropy thus plays an important and sorely neglected role in theory.
\end{abstract}

The understanding of polyelectrolytes is one of the important unresolved problems in polymer physics [1-3]. The presence of charges on the polymer chains presents difficult problems both theoretically and experimentally. Recent application of computer simulations to polyelectrolytes has revealed many interesting results and illuminated various problems with the theoretical approaches [4-7]. References [4-6] treated the complete Coulomb interactions for salt-free polyelectrolytes in solutions. That is, the counterions in the solutions were explicitly simulated, and the full $1 / r$ potential was treated including long-range effects. In contrast, all theoretical approaches use a screened Coulomb or Yukawa pair potential to treat the effective charged monomer interactions [8-12]. In this paper, we report results of simulations of polyelectrolytes in solution with the Coulomb interactions treated at the Debye-Hückel (DH) level. In this way we can determine the utility of the $\mathrm{DH}$ approximation in theory.

The DH approximation is the linearization of the Poisson-Boltzmann equations which are the mean-field equations. Because the DH equation is linear there is an analytic form for the $\mathrm{DH}$ pair potential,

$$
u_{\imath \jmath}(r)=q^{2} k_{\mathrm{B}} T \lambda_{\mathrm{B}} \exp (-\kappa r) / r
$$

where $\lambda_{\mathrm{B}}=e^{2} / \epsilon k_{\mathrm{B}} T$ is the Bjerrum length, $\kappa=\Lambda^{-1}=\left(4 \pi \lambda_{\mathrm{B}} \rho\right)^{1 / 2}$ is the inverse Debye length in a salt-free solution, $\rho$ is the monomer density and $q$ is the monomer charge which is always one electron charge in this work. Theoretical works use the DH potential, since the full

$\left(^{*}\right)$ Author for correspondence (e-mail: kremer@mpip-mainz.mpg.de) 
Coulomb potential is presently intractable as are other approximations such as the nonlinear Poisson-Boltzmann equations for flexible chains. However, the validity of the DH approximation for polyelectrolytes especially in salt-free solution is questionable [13]. The Coulomb interactions for a polyelectrolyte in a salt-free solution tend to be strong in contradiction of the $\mathrm{DH}$ assumption of weak interactions.

In the theory of charged colloids, the Debye potential is often used with success particularly for dilute concentrations, but the bare macroion charge, $Z$, must be renormalized [14]. (Sometimes the Debye screening length is also renormalized.) The physical concept for renormalization of $Z$ is similar to the valence concept for atoms. The tightly bound ("condensed") counterions contribute to neutralizing the bare macroion charge resulting in a smaller effective macroion charge, similar to the core electrons and the nucleus combining to form an ion. The counterions in the interstitial regions determine the interparticle interactions much as valence electrons [15]. The $\mathrm{DH}$ approximation is often valid in the interstitial region, because there the electrostatic potential energy is not large compared to $k T$.

For colloidal systems, the macroion shape is fixed and unimportant, but for polyelectrolytes the shape depends on the electrostatic interactions. The interactions near the polyelectrolyte are essential in determining the chain structure and the interaction energies are large compared to $k T$. Whether a renormalized $\mathrm{DH}$ approach will work is questionable. While some renormalization is at least necessary to improve the results of a $\mathrm{DH}$ based calculations, we performed no renormalization in this paper for two reasons. First of all, in theoretical calculations that use the $\mathrm{DH}$ potential and to which the simulations are compared, no renormalization is performed. Second, and clearly related to the first, there is no definitive renormalization scheme for polyelectrolyte chains.

We use the same basic polymer model used in the earlier polyelectrolyte and neutral polymer simulations $[4-6,16]$. The polymers are modeled as freely-jointed bead-chains. The charged monomers interact via the Debye-Hückel pair potential. The solvent is modeled by a dielectric background. There have been several previous simulations treating a single DH chain [7, 17], revealing severe problems for the weakly charged case. Here, we examine a solution of strongly charged chains and make direct comparisons with our previous simulations that explicitly treated the counterions and the full Coulomb interactions which we will label as the "Coulomb" simulations [4-6].

We performed simulations for the $\mathrm{DH}$ systems with the same parameter values as for the earlier Coulomb systems. The $\mathrm{DH}$ simulations were performed with $N=32$ and 64 beads, and for some densities extended to $N=128$ beads. The number of chains was either 8 or 16 such that the simulation cell length was greater than the average end-to-end distance. Simulations containing 64 chains have been performed for the Coulomb systems with the same results 8 to 16 chains systems. The bond potential is the standard FENE (nonlinear spring) potential with spring constant $k=7 \epsilon / \sigma^{2}$, and maximum extent, $R_{0}=2 \sigma$, where here, as throughout the letter, Lennard-Jones units are used [16]. The average bond length, $b$, is 1.1 $\sigma$. Excluded volume effects among the monomers are included via a purely repulsive Lennard-Jones potential with the cutoff at $2^{1 / 6} \sigma$ and the potential shifted to zero at the cutoff.

The Coulomb coupling strength is determined by the Bjerrum length, $\lambda_{\mathbf{B}}$, which is taken to be the same as in the earlier works $\lambda_{\mathrm{B}}=\sigma / 1.2$. Since we are treating $\mathrm{DH}$ chains it is important that $\lambda_{\mathrm{B}} / b<1$, for larger values lead to significant counterion condensation which cannot be directly treated by the $\mathrm{DH}$ pair potential $[6,10]$.

The dynamics of the system is performed at constant temperature, $T=1.2 \epsilon$, using the Langevin thermostat with damping constant $\Gamma=1 \tau^{-1}$, and time step $0.015 \tau$ [16]. The length of the simulation is such that the chains move at least 10 times the contour length, $L$. For 32 bead chains this required about 300,000 time steps. 


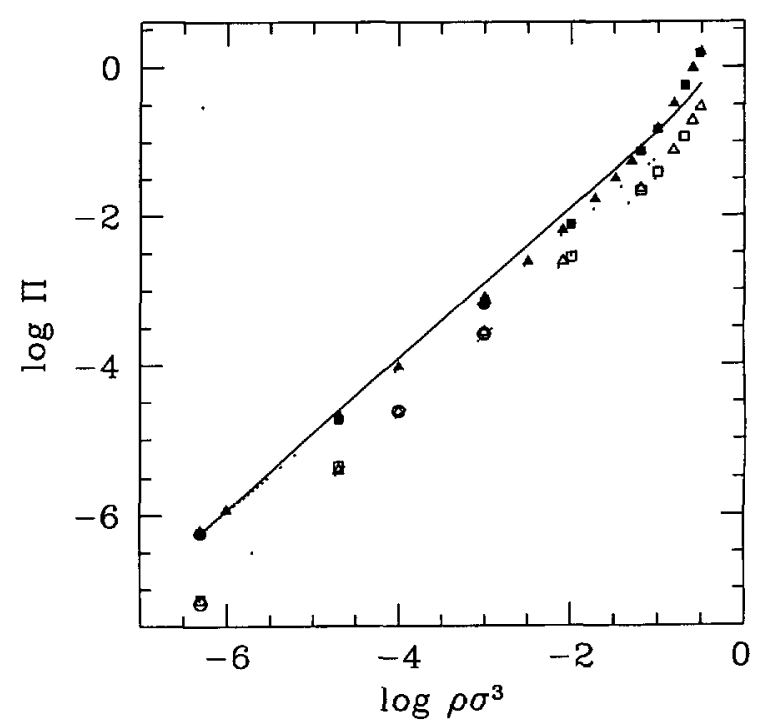

Fig. 1. - The osmotic pressure is plotted as a function of the monomer density on a log-log scale for various chain lengths. The 32,64 and 128 bead chains are represented by triangles, squares and circles, respectively. The solid points represent the Coulomb simulation data and the open points represent the DH simulation data. The solid line represents the $\mathrm{DH}$ data with the counterions' ideal gas term included. The two dotted lines are least square fits to the $N=64$ data in the low density regime. The slopes are 0.93 and 1.07 for the Coulomb and DH data, respectively.

We can map our model onto an experimental system in the following manner. For NaPSS in water, $\lambda_{\mathrm{B}}=7.14 \AA$ which implies from $\lambda_{\mathrm{B}}=0.833 \sigma$ that $1 \sigma=8.57 \AA$. The bond length for NaPSS is $2.5 \AA$. Our charge separation is then $9.43 \AA$ which requires that the fraction of charged sites is $f=0.26$. The simulation bead corresponds to $1 / f$ monomers (charged or not) of the polymer. The bead density of $1 \sigma^{-3}$ corresponds to the monomer concentration $10 \mathrm{M}$, where the factor $1 / f$ has been included. Since only the bead density is used in this article, we use the term "monomer density".

We now discuss the results of the DH simulations comparing to the Coulomb simulations. Three quantities are examined: the osmotic pressure, $\Pi$, the end-to-end distance, $R$, and the single chain structure factor, $S(q)$.

In agreement with experimental results [18], the previous Coulomb simulations [6] found two regimes in the osmotic pressure, $\Pi$. At high densities $\Pi$ scales as $\rho^{9 / 4}$ which is the scaling for semidilute neutral polymers [19]. The neutral scaling holds above the density where $\Lambda=b$ at which the Coulomb interactions are completely screened. At lower densities the Coulomb interaction dominates and the scaling depends on the dimensionless Coulomb strength, $\lambda_{\mathrm{B}} / b$, as well as the chain length [6]. It is important to realize that the two regimes indicate a change in the dominant interaction, not a change in the chain structure which varies continuously throughout both regimes (in strong variance to neutral polymers).

The osmotic pressure calculated in the $\mathrm{DH}$ simulations has some interesting contrasts to the Coulomb simulations. In Figure 1 we plot the $\mathrm{DH}$ and Coulomb osmotic pressures. $\Pi_{\mathrm{DH}}$ and $\Pi_{C}$, respectively. The uncertainty in the pressures is smaller than the points. A wide range of densities is covered. Using the map mentioned above, our lowest densities correspond to $5 \times 10^{-6} \mathrm{M}$ which is really below the physical limit in experiments. Such low densities are 
required for comparison to theoretical limits. The two pressures do not agree because various counterion contributions are missing in $\Pi_{\mathrm{DH}}$. By adding the ideal gas counterion contribution to $\Pi_{\mathrm{DH}}$, we obtain values closer to $\Pi_{\mathrm{C}}$, but they still disagree except at the most dilute densities. Moreover, the scaling behavior at high densities and the crossover to the neutral scaling is altered by the addition of the counterion ideal gas term.

From the dotted lines in Figure 1 the $\Pi_{D H}$ clearly has a different scaling than $\Pi_{C}$ in the low density regime. The line through the $\Pi_{D H}$ data is a least squares fit to the $N=64$ data and has a slope of 1.07. The fit line through the $N=64 \Pi_{\mathrm{C}}$ data has a slope of 0.93 (for more details see also [6]). The value of this slope is $N$-dependent as $\Pi_{\mathrm{C}}$ has a very weak $N$-dependence for $\rho$ in the vicinity of $10^{-3} \sigma^{-3}$ By $\rho=10^{-6} \sigma^{-3}$ this $N$-dependence vanishes as the system approaches the zero density non-interacting limit, $\Pi_{\mathrm{C}}=\dot{k}_{\mathrm{B}} T \rho(1+1 / N)$. In contrast to these results, $\Pi_{D H}$ has no $N$-dependence below $\rho=0.1 \sigma^{-3}$ and it scales as $\rho^{1.07}$. This does not yield the correct non-interacting limit. Even the inclusion of the ideal gas contribution yields an different scaling in the dilute regime than the Coulomb data.

The discrepancies at low densities are in part due to the $\mathrm{DH}$ pair interaction (Eq. (1)) being inaccurate at low densities. As $\rho \rightarrow 0, \kappa \rightarrow 0$, and the $\mathrm{DH}$ pair potential becomes a bare Coulomb potential. For a system of chains this completely neglects the counterion screening of between charged polymers. The counterions around a chain at very low densities will almost completely screen the chains from each other as was found in the Coulomb simulations. Yet, the $\mathrm{DH}$ simulations allow the chains to interact. Ultimately, the zero density limit is the $\mathrm{DH}$ approximation, but this occurs because as $\rho \rightarrow 0$ the average macroion separation, $a$ approaches $\infty$. In other words, the average Coulomb interaction is much less than $k_{\mathrm{B}} T$. One might think that at our low densities the separations are sufficient to yield negligible interactions, but this is grossly wrong because the charge on the chains is large and the interaction depends on the square of this charge. For example, consider $\rho=10^{-6} \sigma^{-3}$ The average chain-chain separation for $N=64$ chains is $a=(\rho / N)^{-1 / 3}=400 \sigma$. The DH pair interaction of two chains at the average separation is

$$
k_{\mathrm{B}} T \lambda_{\mathrm{B}} \frac{N^{2}}{a} \mathrm{e}^{-\kappa a}=7.2 k_{\mathrm{B}} T .
$$

Thus, the chain-chain $\mathrm{DH}$ interactions are several times $k_{\mathrm{B}} T$ even at our very dilute densities! In a similar manner chain-chain quantities as well as the total system quantities in the DH simulations differ from the Coulomb simulations.

The interchain interactions would be better modeled using the $\mathrm{DH}$ potential with a renormalized charge, $Z^{*}[14,15]$. The value of $Z^{*}$ would be much less than $N$, the bare chain charge [15]. This reflects the stronger screening of the counterions than accounted for by the $\mathrm{DH}$ approximation. While a $Z^{*}$ can be chosen to accurately treat the interchain interactions, this choice value will probably be poor for the intrachain interactions. Furthermore, one must determine how to choose the renormalized monomer charge $q^{*}$ given $Z^{*}$. Such an investigation is outside this work.

To see the effect of the DH approximation on the single chain structure, we examine the square end-to-end distance, $\left\langle R^{2}\right\rangle$, as a function of density. Figure 2 shows that $\left\langle R^{2}\right\rangle$ for $N=64$ tends to be larger at low densities for the $\mathrm{DH}$ simulations and is smaller at high densities. This seems to be a generic feature for the various $N$. The crossover occurs near the overlap density of the chains, $\rho^{*} \approx 0.01 \sigma^{-3}[6]$. For $N=64$ the DH values differ from the Coulomb value by 0 $10 \%$. The uncertainty in the $\mathrm{DH}$ values is smaller than the points in the figure. For our values of $N$, the difference between Coulomb and $\mathrm{DH}$ is not very large, although the difference is beyond the uncertainty particularly for $N=64$ at the lowest densities. The density dependence for both methods should be similar, since $R$ must vary monotonically between the neutral limit and the "rod" limit. Because the DH approximation underestimates the counterion density 


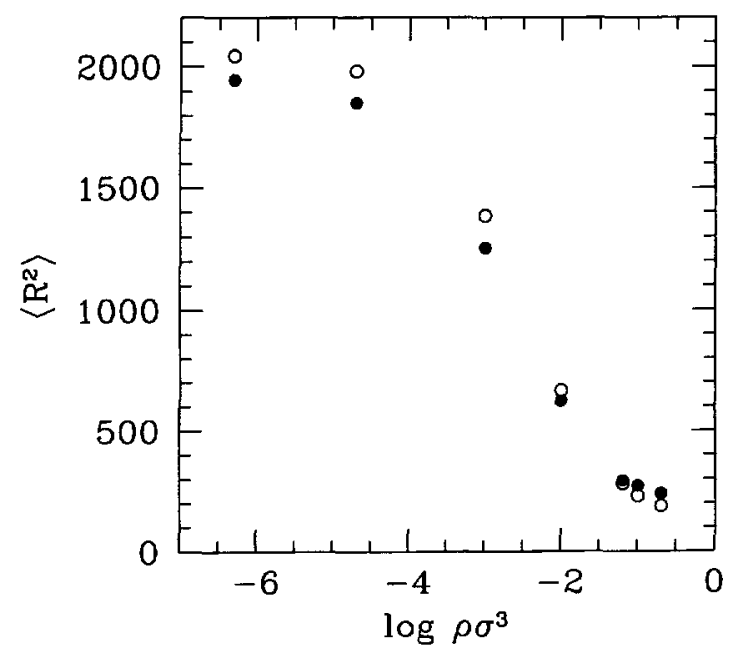

Fig. 2. - The square end-to-end distance as a function of density is plotted at $N=64$ for Coulomb data (solid) and DH data (open).

near the chain which screens the monomer interactions $[6,14]$, the Coulomb chains should be more coiled. This effect can be seen in Figure 2 in the dilute regime.

In the semidilute regime, the $\mathrm{DH}$ chains become more coiled than the Coulomb chains in contrast to the dilute regime. The relative importance of various interactions changes upon entering the semidilute regime. In the dilute regime, the chain structure is predominantly determined by the intrachain interactions, but in the semidilute regime the interchain interactions now effect the chain structure as well. The interactions can be thought of on a segment-segment basis where a segment is the part of a chain between two overlap points. Two segments need not be part of the same chain. The Coulomb interactions between segments are relatively short-ranged and not in the realm where the $\mathrm{DH}$ approximation is valid. The smaller $\left\langle R^{2}\right\rangle$ for the $\mathrm{DH}$ simulations suggests that the $\mathrm{DH}$ potential is too weak at these densities. At high densities, the $\mathrm{DH}$ approximation for simple electrolytes is known to yield large values for the correlation function between like charged ions particularly at short separations [20]. Thus, the monomers for the DH simulations are closer together yielding a smaller $\left\langle R^{2}\right\rangle$.

One of the important results of the Coulomb simulations is also true in the $\mathrm{DH}$ simulations. Namely, the chains contract significantly below $\rho^{*}$ in contrast to all theoretical predictions $[21,22]$. At dilute densities, entropy and Coulombic interactions compete to determine the chain structure. Entropy favors a coiled structure with many equivalent states, but Coulombic interactions favor a rodlike structure which has low entropy. The contraction below $\rho^{*}$ implies that entropy is not dominated by the Coulomb interaction and cannot be neglected ( $c f .[12]$ ). The basis of much early work in polyelectrolytes treated the polyelectrolyte chain as a rigid rod completely neglecting entropy [8,9]. Clearly, this treatment must be altered as also found in recent single chain simulations of weakly charged DH chains [7]. There it was found that the standard DH weakly charged chains does not provide a useful model.

A more detailed analysis of the structure is given by the single chain structure factor,

$$
S(q)=\left\langle\frac{1}{N}\left|\sum_{j=1}^{N} \mathrm{e}^{\imath \mathbf{q} \mathbf{r}_{J}}\right|^{2}\right\rangle,
$$




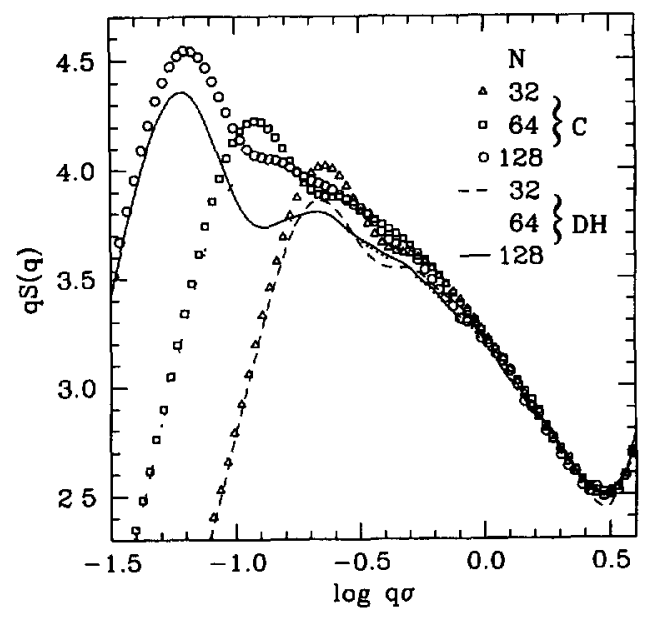

Fig. 3. - At $\rho=0.001 \sigma^{-3}, q S(q)$ is plotted for both the Coulomb (C) and Debye-Hückel (DH) simulations.

where the average is over time, all chains and angles, and $r_{3}$ is the position of the $j$ th monomer. Figure 3 shows $q S(q)$ for the $\mathrm{DH}$ and Coulomb simulations at $N=32,64$ and 128 for $\rho=$ $0.001 \sigma^{-3}$ which is a dilute density for all $N$. We choose to plot $q S(q)$ instead of the usual Kratky quantity of $q^{2} S(q)$, because the chains are uncoiled especially at dilute densities with a scaling of $S(q) \sim q^{-1 / \nu}$ with $\nu \approx 0.9$.

Since the density is dilute and the $\mathrm{DH}$ chains are more elongated: $S_{\mathrm{DH}}(q) \leq S_{\mathrm{C}}(q)$ for $2 \pi / b \gtrsim$ $q$. In terms of the scaling exponent, $\nu$, the $\mathrm{DH}$ chains have $\nu$ closer to 1 than the Coulomb chains. However, in neither case is there a rodlike regime. For distances $\xi=2 \pi / q \gtrsim 6 \sigma$ the $\mathrm{DH}$ and Coulomb $S(q)$ differ significantly in Figure 3. The DH chains on these length scales are more elongated than the Coulomb chains. Also visible in Figure 3 is the stronger presence of oscillations in $S(q)$ at low $q$ in the DH data. Particularly in the $N=128$ data, the $S_{\mathrm{C}}(q)$ shows no oscillation down to $\log q \sigma=-1$, whereas the $S_{\mathrm{DH}}(q)$ shows oscillations beginning at $\log q \sigma=-0.6$. Since the $\mathrm{DH}$ chains are more expanded, their extension displays smaller relative fluctuations. As expected this shows up in more pronounced "cutoff" fluctuations in $S(q)$.

The functional form and density dependence of $S_{\mathrm{DH}}(q)$ is similar to $S_{\mathrm{C}}(q)$. In contrast to theoretical predictions [21-23], $S_{\mathrm{DH}}(q)$ does not exhibit the predicted two $q$-regimes: $\nu=1$ for $2 \pi / b>q>2 \pi / L_{\mathrm{p}}$, and $\nu=1 / 2$ or $3 / 5$ depending on the solvent quality for $q>2 \pi / L_{\mathrm{p}}$, where $L_{\mathrm{p}}$ is the persistence length: Instead as found in the Coulomb simulations, $\nu$ varies continuously although with a small difference from the Coulomb value. At high $q$ between $2 \pi / 6 \sigma$ and $2 \pi / 2 b, S_{\mathrm{DH}}(q)$ does not possess the density independence found in $S_{\mathrm{C}}(q)$. Instead the value of $\nu$ in this regime is also continuously varying. This suggests that the discreteness of the counterions may contribute to the density independence found in the Coulomb simulations for this $q$-regime.

In conclusion, we have performed simulations of polyelectrolytes in salt-free solutions using the Debye-Hückel approximation for the charged interactions. These simulations show some of the limitations of this approximation. Because the DH interchain interactions are incorrect, not only is the magnitude of the osmotic pressure incorrect, but also the density dependence is wrong. The incorrect interchain interactions limits the $\mathrm{DH}$ model to single chain properties, 
but there too the $\mathrm{DH}$ model has weaknesses. The end-to-end length is overestimated at low densities, although the qualitative density dependence is the same as for Coulomb chains. Two important points are that the $\mathrm{DH}$ chains contract below the overlap density in contradiction of all theories, and the single chain structure factor does not have the predicted form. These results clearly indicate that entropy plays an important role for the $\mathrm{DH}$ chains. While the $\mathrm{DH}$ approximation has its faults and cannot, for example, address the effect of counterion condensation on chain structure [6], a correct theoretical calculation of the structure of DH chains has yet to be done. Clearly, theory must be improved to handle competition between entropy and the charged interactions beyond the level of the $\mathrm{DH}$ approximation.

\section{Acknowledgments}

We would like to acknowledge a large grant of computer time from the Höchstleistungsrechenzentrum HLRZ in Jülich, Germany, within the Disordered Polymers Project and support from a NATO Travel Grant. K.K. acknowledges the hospitality of the IFF of the Forschungszentrum Jülich, Germany.

\section{References}

[1] Macro-ion Characterization: From Dilute Solutions to Complex Fluids, K. Schmitz Eds. (American Chemical Society, Washington, D.C., 1994).

[2] Förster S. and Schmidt M., Adv. Polymer Scr. 120 (1995) 51.

[3] Barrat J.-L. and Joanny J.-F., Adv. Chem. Phys. XCIV (1966) 1.

[4] Stevens M. and Kremer K., Macromolecules 26 (1993) 4717.

[5] Stevens M. and Kremer K., Phys. Rev. Lett. 71 (1993) 2228.

[6] Stevens M. and Kremer K., J. Chem. Phys. 103 (1995) 1669.

[7] Micka U. and Kremer K., Phys. Rev. E, in press (1996).

[8] Odijk T., J. Polym. Scr., Polym. Phys. Ed. 15 (1977) 477.

[9] Skolnick J. and Fixman M., Macromolecules 10 (1977) 944.

[10] Manning G., Q. Rev. Biophys. 11 (1978) 179.

[11] Witten T. and Pincus P., Europhys. Lett. 3 (1987) 315.

[12] Barrat J.-L. and Joanny J.-F., Europhys. Lett. 3 (1993) 343.

[13] Rice S.A. and Nagasawa M., Polyelectrolyte solutions, a theoretical introduction (Academic Press, New York, 1961).

[14] Alexander S., Chaikin P.M., Grant P., Morales P.J., Pincus P. and Hone D., J. Chem. Phys. 80 (1984) 5776.

[15] Stevens M., Robbins M. and Falk M., J. Chem. Phys. 99 (1995) 99.

[16] Dünweg B. and Kremer K., Phys. Rev. Lett 66 (1982) 2996.

[17] Dünweg B., Stevens M. and Kremer K.. in Monte Carlo and Molecular Dynamics Simulations in Polymer Science, K. Binder Eds. (Oxford, New York, 1995).

[18] Wang L. and Bloomfield V., Macromolecules 23 (1990) 804.

[19] de Gennes P.-G., Scaling Concepts in Polymer Physics (Cornell University, Ithaca, NY, 1979).

[20] Card D.N. and Valleau J.P., J. Chem. Phys. 52 (1970) 6232.

[21] de Gennes P.-G., Pincus P. and Velasco R., J. Phys. France 37 (1976) 1461.

[22] Odijk T., Macromolecules 12 (1979) 688.

[23] Hayter J., Janninck G., Brochard-Wyart F. and de Gennes P.-G., J. Phys. France Lett. 41 (1980) 451. 